\title{
Controle de infecção hospitalar em unidade de terapia intensiva: estudo retrospectivo
}

\section{Hospital infection control in intensive care unit: retrospective study}

\author{
Patricia Terron Ghezzi M. Abegg ${ }^{1}$; Ligiane de Lourdes da Silva ${ }^{2}$
}

\begin{abstract}
Resumo
Este estudo teve como objetivo verificar a ocorrência de microorganismos isolados de pacientes hospitalizados durante as etapas de implantação da Comissão de Controle de Infecção Hospitalar (CCIH) em uma Unidade de Terapia Intensiva de um hospital privado na cidade de Toledo, Paraná. Trata-se de um estudo retrospectivo, durante o período de fevereiro de 2007 a março de 2008. Do total de internamentos, 37 pacientes apresentaram infecção, $73 \%$ do sexo masculino, com média de idade de 71 anos. Na primeira fase de coleta de dados, antes da implantação da CCIH, 12 pacientes apresentaram culturas positivas e Pseudomonas aeruginosa foi o microorganismo mais isolado, com incidência de 30,77\%, seguido de Staphylococcus epidermidis, 23,08\%, seguidos por outros sete microorganismos com incidências inferiores. Na segunda fase, durante o início das atividades, 10 pacientes apresentaram infecção, com crescimento de Pseudomonas aeruginosa, 30\%, Klebsiella sp., 15\%, Staphylococcus aureus, Klebsiella pneumoniae, Staphylococcus epidermidis, Enterobacter cloacae, 10\%, e Streptococcus Gama Hemolíticos, Escherichia coli, Enterobacter sp., 5\%. Com as normas da CCIH sendo ativamente empregadas na terceira fase da coleta de dados, percebe-se que essa relação se distancia, resultando em cinco pacientes com infecção, sendo Pseudomonas aeruginosa, $75 \%$ e Staphylococcus epidermidis e Streptococcus Beta Hemolíticos, 12,5\%. Aproximadamente um ano após o início das atividades da $\mathrm{CCIH}$, observa-se que na quarta fase, seis pacientes apresentaram infecção, com Pseudomonas aeruginosa, 54,5\% e Enterobacter aerogenes, 45,5\%. A informação, o treinamento, o comprometimento e conscientização da equipe médica e colaboradores, associados ao uso diário das normas preconizadas pela Comissão de Controle de Infecção Hospitalar são ferramentas imprescindíveis para tornar possível a obtenção de resultados significativos e satisfatórios na diminuição dos casos de infecção hospitalar, melhorando a qualidade de assistência à saúde.
\end{abstract}

Palavras-Chave: Controle de infecção hospitalar. Pseudomonas aeruginosa. Staphylococcus epidermidis. Unidade de terapia intensive.

\begin{abstract}
This study aimed at verifying the occurrence of isolated microorganisms of hospitalized patients during the stages of implantation of the Hospital Infection Control Commission (HICC) in an Intensive Care Unit at a private hospital in the city of Toledo, Paraná. It is about a retrospective study, during the period of February/ 2007 to March/2008. Of the total internments, 37 patients presented positive cultures and Pseudomonas aeruginosa was the most isolated microorganism, with $30.77 \%$ incidence, followed by Staphylococcus epidermidis, $23.08 \%$, followed by other seven microorganisms with inferior incidences.
\end{abstract}

\footnotetext{
1 Farmacêutica Bioquímica - Especialista em Microbiologia Aplicada. Especialização em Análises Clínicas e Toxicológicas FAG. R: Raimundo Leonardi n³0, Apto. 03, tel: (45) 30541882. Toledo - Paraná, Brasil. Email: oipatty@hotmail.com.

2 Professor Assistente do Centro de Ciências Médicas e Farmacêutica - CCMF. Universidade Estadual do Oeste do Paraná UNIOESTE. Rua: Universitária no 2069, tel: (45) 3200-3255. Caixa Postal: 711 CEP: 85814-110. Cascavel - Paraná, Brasil. Email: ligianes@gmail.com.
} 
In the second phase, during the beginning of the activities, 10 patients presented infection, with the growth of Pseudomonas aeruginosa, 30\%, Klebsiella sp., 15\%, Staphylococcus aureus, Klebsiella pneumoniae, Staphylococcus epidermidis, Enterobacter cloacae, 10\%, and Streptococcus hemolytic gamma, Escherichia coli, and Enterobacter sp., 5\%. With the CCIH norms being actively used in the third phase of the data collection, it is possible to realize that this relation distances itself, resulting in five patients with infection, being Pseudomonas aeruginosa, 75\% and Staphylococcus epidermidis and Streptococcus hemolytic beta, $12.5 \%$. About one year after the beginning of the activities of the HICC, it is observed that in he fourth phase, six patients presented infection, with Pseudomonas aeruginosa,54.5\% and Enterobacter aerogenes, $45.5 \%$. The formation, the training, the commitment and collaborating awareness of the medical staff, associated with the daily use of the norms praised by the hospital Infection Control Commission, are essential tools to enable the satisfactory and meaningful result in the reduction of the cases of hospital infection, improving the quality of health assistance.

Keywords: Hospital infection control. Pseudomonas aeruginosa. Staphylococcus epidermidis. Intensive care unit.

\section{Introdução}

Pacientes internados em instituições de saúde estão expostos a uma ampla variedade de microorganismos patogênicos, principalmente em Unidades de Terapia Intensiva (UTI), onde o uso de antimicrobianos potentes e de amplo espectro é regra, e os procedimentos invasivos são de rotina (FERRAZ, 1987).

Segundo conceito do Ministério da Saúde (MS), na portaria $n^{\circ} 2.616$ de 12.05.1998, as infecções hospitalares restringem-se àquelas adquiridas após a admissão do paciente na unidade hospitalar e que se manifestam durante a internação ou após a alta, quando puder ser relacionada com a internação ou procedimentos hospitalares. Essa definição não indica o agente infeccioso específico, nem localização particular da infecção, mas apenas define o espaço físico onde ela se inicia. Seu estudo ainda é um tema novo das ciências médicas (BRASIL, 1998; CEPEDA et al., 2005; BRASIL, 1985a; BRASIL, 1985b).

A infecção é uma entidade clínica de múltiplos fatores envolvidos, e a necessidade de reduzir e controlar sua incidência determina a aplicação de medidas preventivas, educacionais e de controle epidemiológico que visam, por meio de um processo de conscientização coletiva, a levar a taxas de infecção para limites aceitáveis para o tipo de clientela e de procedimentos realizados em cada hospital (FERRAZ et al., 2001).

No Brasil, após a promulgação da portaria 196/83, o Ministério da Saúde elaborou um estudo em que foram avaliados 8.624 pacientes com mais de 24 horas de internação, cujo tempo médio de permanência foi de 11,8 dias. O número de pacientes com infecção hospitalar encontrado foi 1.129 , com taxa de pacientes com infecção hospitalar de 13\%. Os maiores índices de infecção (18.4\%) foram obtidos em hospitais públicos e os menores (10\%) em hospitais privados sem fins lucrativos. A região Sudeste apresentou $16,4 \%$ dos casos de pacientes com quadro de infecção hospitalar, seguida do Nordeste com 13,1\%, Norte com 11,5\%, Sul com 9\% e Centro Oeste com 7,2\%. Diante destes dados, as infecções hospitalares são apontadas como um dos mais importantes riscos aos pacientes hospitalizados. Isso justifica a inclusão dos índices de infecção hospitalar como um parâmetro de controle da qualidade do serviço prestado por um hospital (ANDRADE; ANGERAMI, 1999; PRADE; OLIVEIRA; RODRIGUES, 1995).

Pacientes críticos hospitalizados em Unidade de Terapia Intensiva são mais vulneráveis à infecção hospitalar em comparação com as demais unidades, por seu alto risco de infecção e alta complexidade no atendimento. $\mathrm{O}$ uso de procedimentos mais invasivos, além da utilização de antimicrobianos de amplo espectro na rotina, faz surgir bactérias cada vez mais resistentes. Esses pacientes críticos têm de 
cinco a dez vezes maior probabilidade de contrair uma infecção hospitalar, pois estão frequentemente expostos aos fatores de risco: cirurgias complexas, drogas imunossupressoras, antimicrobianos, interação com a equipe de saúde, fômites, entre outras, que podem resultar em cerca de $20 \%$ do total de infecções do hospital. O risco de infecção é diretamente proporcional à gravidade da doença, condições nutricionais, natureza dos procedimentos diagnósticos e/ou terapêuticos, bem como, ao tempo de internação, dentre outros aspectos (COUTO; PEDROSA; NOGUEIRA, 2003; PILONETTO et al., 2004; VINCENT, 2003).

Além disso, as infecções hospitalares influenciam drástica e diretamente o período de hospitalização e nos índices de morbimortalidade, repercutindo nos custos, considerando o prolongamento da internação, o consumo de antibióticos, exames laboratoriais e os gastos com isolamento. Estima-se que o aumento da resistência bacteriana nos Estados Unidos da América tem provocado um custo anual entre 100300 milhões de dólares (ÉVORA; ALMEIDA, 1983; WOJKOWSKA-MACHJ, 2006).

Nenhum hospital está livre das infecções adquiridas durante a internação, uma vez que nenhuma intervenção médica disponível, no momento, é capaz de erradicá-las, porém a diminuição no número de casos, ou seja, o controle do agravo é possível, e constitui o objetivo dos vários programas de controle existentes em diversos países. No sentido de identificar e utilizar estratégias de controle comprovadamente eficazes, o governo, em conjunto com a Agência Nacional de Vigilância Sanitária (ANVISA), buscou desenvolver um conjunto de ações deliberadas e sistemáticas, com vistas à redução da incidência e gravidade das infecções hospitalares, que pudesse ser utilizada por todos os profissionais de saúde envolvidos na estrutura da assistência hospitalar. Isso faz com que essas ações fossem também responsáveis pela estrutura, processo e resultados, e disso resultaram os Programas de Prevenção e Controle de Infecção Hospitalar (BRASIL, 1998).
No Brasil, entre aproximadamente 5 e $15 \%$ dos pacientes hospitalizados e 25 a $35 \%$ dos pacientes admitidos em Unidades de Terapia Intensiva adquirem infecção hospitalar, sendo ela a quarta causa de mortalidade, porém os dados sobre infecção hospitalar são pouco divulgados ou antigos. Além disso, esses dados não são consolidados por muitos hospitais, o que dificulta o conhecimento da dimensão do problema no país. Sabe-se que as doenças infecciosas matam de 17 a 20 milhões de pessoas por ano no mundo, além disso, cerca de 10 milhões adquirem infecção hospitalar e, desse universo, quase 300 mil morrem (BUCHALLA; WALDMAN; LAURENTI, 2003; CAO et al., 2004; DIENER; COUNTINHO; ZOCCOLI, 1996; TURRINI, 1996; VINCENT, 2003; ASSOCIAÇÃO PAULISTA DE ESTUDOS E CONTROLE DE INFECÇÃO HOSPITALAR, 2005).

A captação de dados sobre o controle da freqüência e distribuição das infecções hospitalares é efetuada pela Vigilância Epidemiológica municipal, que o realiza a partir da coleta de informações e da notificação controlada, preenchida pelos médicos responsáveis pelo atendimento após alta ou óbito do paciente. O hospitalé posteriormente fiscalizado pela Vigilância Sanitária, a qual não somente inspeciona como também deve prestar cooperação técnica aos hospitais, orientando para o exato cumprimento e aplicação das diretrizes estabelecidas pela legislação sanitária. Essa metodologia é considerada deficiente por vários autores, devido a sua baixa sensibilidade e incapacidade de detectar situações anormais, já que a coleta e a análise dos dados, como citado acima, é retrospectivo, ou seja, após alta ou óbito do paciente. Por ainda estar em fase de implantação, o processo de padronização dos serviços ainda se mostra deficiente, o que dificulta ainda mais as inspeções da Vigilância Sanitária (CARDO, 1987; ÉVORA; ALMEIDA, 1983; GIUNTA; LACERDA, 2006).

A taxa de prevalência de infecção hospitalar também depende da utilização correta das técnicas de vigilância epidemiológica pelos colaboradores, 
dos critérios de diagnósticos, capacitação dos profissionais envolvidos, e dos fatores de risco intrínsecose extrínsecos presentes numa determinada unidade em um determinado tempo. No entanto, a metodologia baseada na busca ativa de casos, realizada pelas Comissões de Controle de Infecção Hospitalar (CCIH), grupo especialmente treinado em cada hospital para o controle da infecção, vem sendo apontada, na literatura americana e inglesa, como o método de escolha para coleta de dados para infecção hospitalar (COUTO; PEDROSA; NOGUEIRA, 2003; GIUNTA; LACERDA, 2006).

A criação e o funcionamento das $\mathrm{CCIH}$ representam um progresso na organização da estrutura hospitalar para a diminuição de múltiplos problemas, como a necessidade de se reduzir e controlar taxas de infecções, o que determinou a aplicação de medidas preventivas, educacionais e de controle epidemiológico, que visam, através de um processo de conscientização coletiva, a levar as taxas de infecção para limites aceitáveis (FERRAZ, 1987).

$\mathrm{Na}$ era pré-antibiótica predominavam as bactérias Streptococcus pyogenes e Staphylococcus aureus como causadoras de infecção hospitalar; contudo, com a introdução da penicilina e a pressão seletiva exercida pelos antimicrobianos, Staphylococcus aureus passou a ser o principal microrganismo associado a epidemias. Em um atual estudo realizado em uma UTI de Santa Catarina, $P$. aeruginosa e Acinetobacter sp. foram identificados como microrganismos de maior ocorrência entre os isolados de casos de infecção hospitalar em UTI (FERNANDES; RIBEIRO FILHO; BARROSO, 2000).

Gales et al. (2001) realizaram um estudo com 70.067 amostras isoladas de pacientes admitidos em hospitais de cinco diferentes áreas geográficas mundiais e verificaram, de acordo com um Programa para Avaliação de Resistência Antimicrobiana, a maior prevalência de infecções causadas por $P$. aeruginosa na América Latina e em regiões da Ásia
(11,4\% do total isolado em cada região), do que na Europa (9,3\%), Estados Unidos da América (8,7\%) e Canadá $(8,6 \%)$. No caso do Brasil, a resistência bacteriana de patógenos hospitalares é um sério problema. A Polimixina, um medicamento altamente tóxico, que está em uso desde a década de 80 para tratamento de infecções causadas por bactérias Gram Negativas, é ainda utilizado para tratar infecções causadas por Pseudomonas aeruginosa, aumentando as chances de multirresistência bacteriana (GALES et al., 2001; LEVIN et al., 1999; ASSOCIAÇÃO PAULISTA DE ESTUDOS E CONTROLE DE INFECÇÃO HOSPITALAR, 2005).

Os agentes infecciosos mais comumente isolados em UTIs são o Staphylococcus epidermidis e Staphylococcus aureus. Staphylococcus epidermidis está presente na pele de indivíduos saudáveis ou não, e pode ser introduzido na UTI por pacientes ou membros colaboradores da saúde, causando infecções oportunistas durante e/ou após procedimentos invasivos. A patogenicidade de Staphylococcus epidermidis está associada à sua resistência a antibióticos, produção de enzimas invasivas, formação de biofilme e essa é uma situação particularmente séria, uma vez que essa bactéria está frequentemente ligada com a colonização de cateteres e implantes, além de ser o agente etiológico de várias infecções nosocomais, como bacteremias, infecções urinárias, endocardites, entre outras (MICHELIM et al., 2005).

O objetivo desse trabalho é verificar a ocorrência de microorganismos isolados de pacientes hospitalizados durante as etapas de implantação da Comissão de Controle de Infecção Hospitalar (CCIH) em uma Unidade de Terapia Intensiva de um hospital privado na cidade de Toledo, Paraná, para assim, avaliar os resultados dos métodos atualmente adotados pela $\mathrm{CCIH}$.

\section{Material e métodos}

Trata-se de um estudo retrospectivo realizado 
em uma Unidade de Terapia Intensiva (UTI), especializada em pacientes com cardiopatias, anexa a um hospital privado na cidade de Toledo, Paraná. Essa unidade possui sete leitos e admite pacientes com idade superior a 14 anos e em situação crítica e semi-crítica. Os dados foram analisados de acordo com as informações contidas nas fichas de controle de infecção, que são anexadas aos prontuários dos pacientes, que, após a alta ou óbito, são encaminhadas à Comissão de Controle de Infecção Hospitalar (CCIH) do hospital, durante o período de fevereiro de 2007 a junho de 2008, após aprovação pelos responsáveis e o Comitê de Ética.

A pesquisa foi realizada em quatro fases. A primeira fase de coleta de dados ocorreu entre os meses de fevereiro e setembro/2007, antes da implantação das normas preconizadas pela Comissão de Controle de Infecção Hospitalar. A segunda ocorreu entre setembro e dezembro de 2007, com o início da execução e adaptação dos colaboradores às novas regras. A terceira etapa foi desenvolvida entre os meses de dezembro de 2007 a março de 2008, quando a coleta de dados aconteceu durante a continuação da implantação das normas e adicional treinamento e aperfeiçoamento de colaboradores para as novas normas e rotinas preconizadas pela $\mathrm{CCIH}$ e, finalmente, a coleta de dados da quarta fase foi realizada entre março e junho de 2008, durante o processo de manutenção das normas.

As coletas do material biológico para as culturas eram realizadas de acordo com a solicitação do médico plantonista, o qual também definia a região, o material e o número de coletas a serem realizadas. A identificação das bactérias foi realizada pelo Laboratório Toledo de Análises Clínicas, que presta serviços terceirizados ao hospital no qual se encontra a UTI em questão, segundo os métodos convencionais de isolamento e identificação bacteriana padronizada pelo NCCLS - National Committe for Clinical Laboratory Standarts. Só foram consideradas as culturas com resultados positivos para algum microorganismo, independente do material (BAUER; KIRBY; SHERRIS, 1966;
NATIONAL COMMITTEE FOR CLINICAL LABORATORY STANDARDS, 2002).

\section{Resultados e discussão}

Do universo das culturas solicitadas de diferentes tipos de secreção dos pacientes internados na UTI cardíaca, 65 resultaram em pelo menos um agente etiológico isolado de 37 pacientes, com idade média de 71 anos, $73 \%$ do sexo masculino. Os microorganismos com maior incidência foram Pseudomonas aeruginosa/sp. e Staphylococcus epidermidis, presentes em 25 e 9 culturas, respectivamente. Na primeira fase de coleta de dados, foram analisados 12 pacientes, com 26 culturas positivas (Figura 1), na segunda fase 10 pacientes, também apresentando 26 culturas positivas (Figura 2).

As infecções causadas por esta espécie de Pseudomonas geralmente são de origem hospitalar. Sabe-se que durante as últimas quatro décadas a incidência por Psedomonas aeruginosa foi responsável por $10 \%$ de todas as infecções nosocomiais. Ela possui características associadas à sua resistência natural, ao grande número de antibióticos empregados (cepas multiresistentes) e antisépticos, o que a torna uma importante causa de infecções epidêmicas e endêmicas, sendo uma das mais frequentes em infecções hospitalares. As manifestações clínicas mais prevalentes ocorrem em: pneumonias, infecções de cateter vascular central, infecção de trato urinário, dentre outras. Quase todos os equipamentos e materiais hospitalares, principalmente os com componentes líquidos, podem servir de reservatório para Pseudomonas ssp., o que, associado a sua multirresistência, pode facilitar a sua disseminação para todo o hospital e para a comunidade extra-hospitalar em transferências de pacientes ou no pós-alta. FILE JUNIOR et al (1999) comprovou que as bactérias Gram-negativas (Escherichia coli, Klebsiella sp., Enterobacter sp.), responsáveis até então pelas infecções pós cirúrgicas, estão dando lugar à bactérias Gram- 
positivas, como Pseudomonas sp. em infecções hospitalares (ANDRADE; LEOPOLDO; HAAS, 2006; FILE JUNIOR, 1999; LEVIN; ARRUDA; OLIVEIRA, 2005; LIMA, 2006; ZAVASCKI; CRUZ; GOLDANI, 2005).

Entre as infecções hospitalares, as sepses por Staphylococcus aureus são responsáveis por elevada morbidade e mortalidade, porém, o Staphylococcus epidermidis é o agente infeccioso mais comumente isolado em UTIs, por estar presente na pele de pessoas saudáveis (microorganismo comensal) responsável então por causar grandes infecções oportunistas, já que pode ser transmitido pelos agentes de saúde durante e após procedimentos de rotina ou mais invasivos. É o agente mais frequentemente encontrado na colonização de implantes e cateteres. Sabe-se que a sua patogenicidade está associada a resistência a antimicrobianos, o que resulta em um problema maior, uma vez que o número de antibióticos viáveis para o tratamento dessas cepas multirresistentes é mínimo. Michelim et al. (2005) evienciaram a presença de Staphylococcus epidermidis com atividades hemolítica, proteolítica e com formação de biofilme em isolados clínicos e controles, o que certamente indica uma maior incidência de cepas com potencial patogênico no ambiente hospitalar (BOYCE, 1999; CAVALCANTI; VALENCIA; TORRES，2005; CEPEDA，2005; GRANSDEN; EYKYN; PHILLIPS, 1984; MICHELIM et al., 2005; MOREIRA et al., 1998; MYLOTTE.; McDERMOTT; SPOONER, 1987; WATANAKUNAKORN et al., 1987).

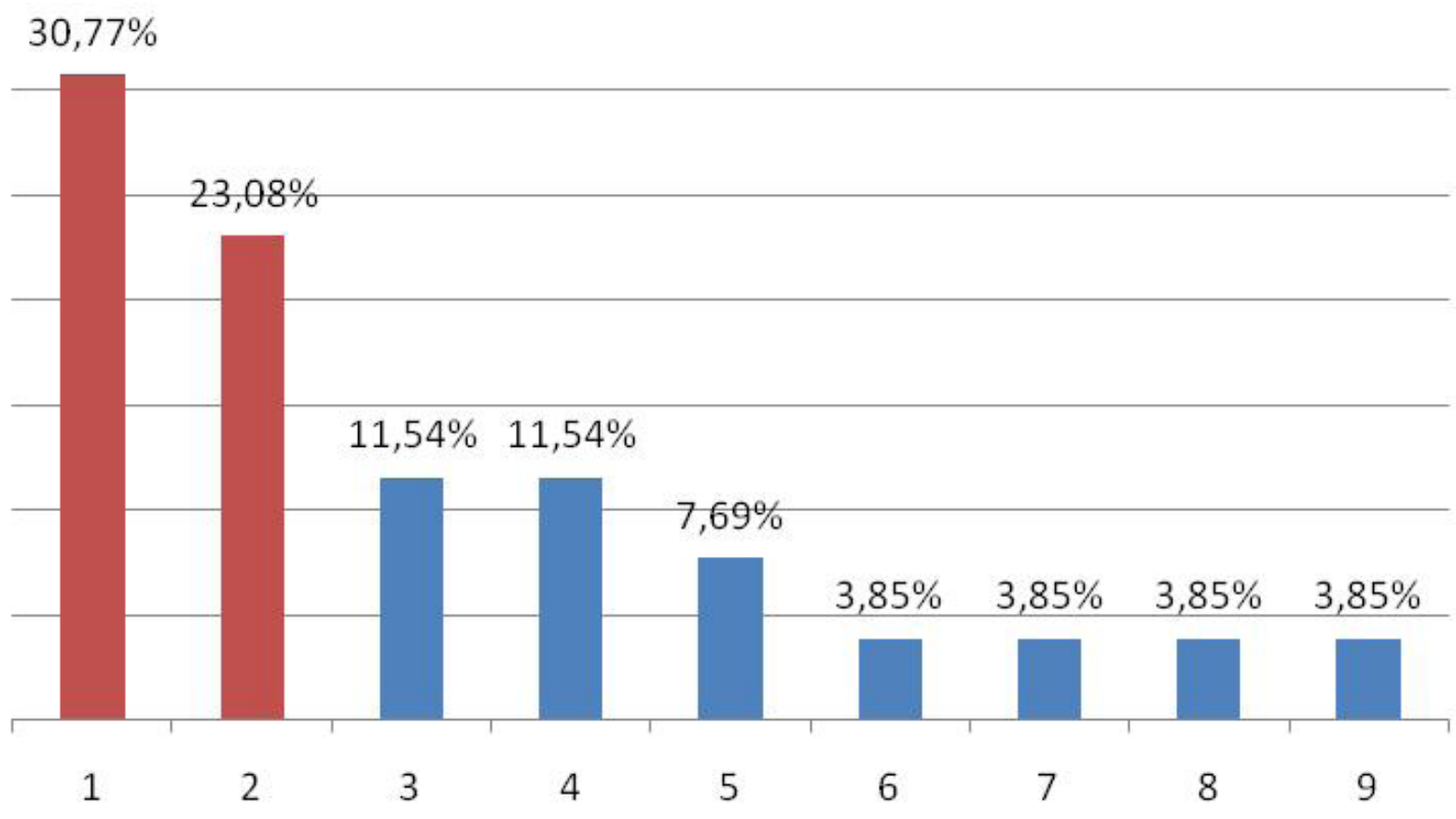

Figura 1. Ocorrência de microorganismos isolados de pacientes antes da implantação das normas preconizadas pela Comissão de Controle de Infecção Hospitalar, durante o período de fevereiro a setembro de 2007, Toledo, Paraná. Total de culturas positivas: 26. $1=$ Pseudomonas aeruginosa; 2 = Staphylococcus epidermidis; 3 = Enterobacter $\mathrm{sp}$; $4=$ Streptococcus sp.; 5 = BGN Não-fermentador; $6=$ Escherichia coli $; 7=$ Candida $\mathrm{sp}$.; $8=$ Klebsiella pneumoniae; $9=$ Streptococcus gama-hemolítico. 
Na segunda fase, de setembro a dezembro/2007, a coleta de dados aconteceu durante o início da implantação e treinamento de colaboradores para as novas normas e rotinas preconizadas pela $\mathrm{CCIH}$, não havendo grande diminuição no número de culturas positivas. De acordo com a figura 2 , houve uma pequena diminuição nas culturas positivas para Staphylococcus epidermidis (10\%) e o número de culturas com crescimento de Pseudomonas aeruginosa praticamente não se alterou (30\%). Se considerarmos o tempo da primeira fase de análises (sete meses) e o compararmos ao desta fase (três meses), observamos que há um aumento de culturas positivas em relação ao tempo, o que é altamente preocupante, e mostra-nos a necessidade urgente de medidas preventivas contra as infecções hospitalares.

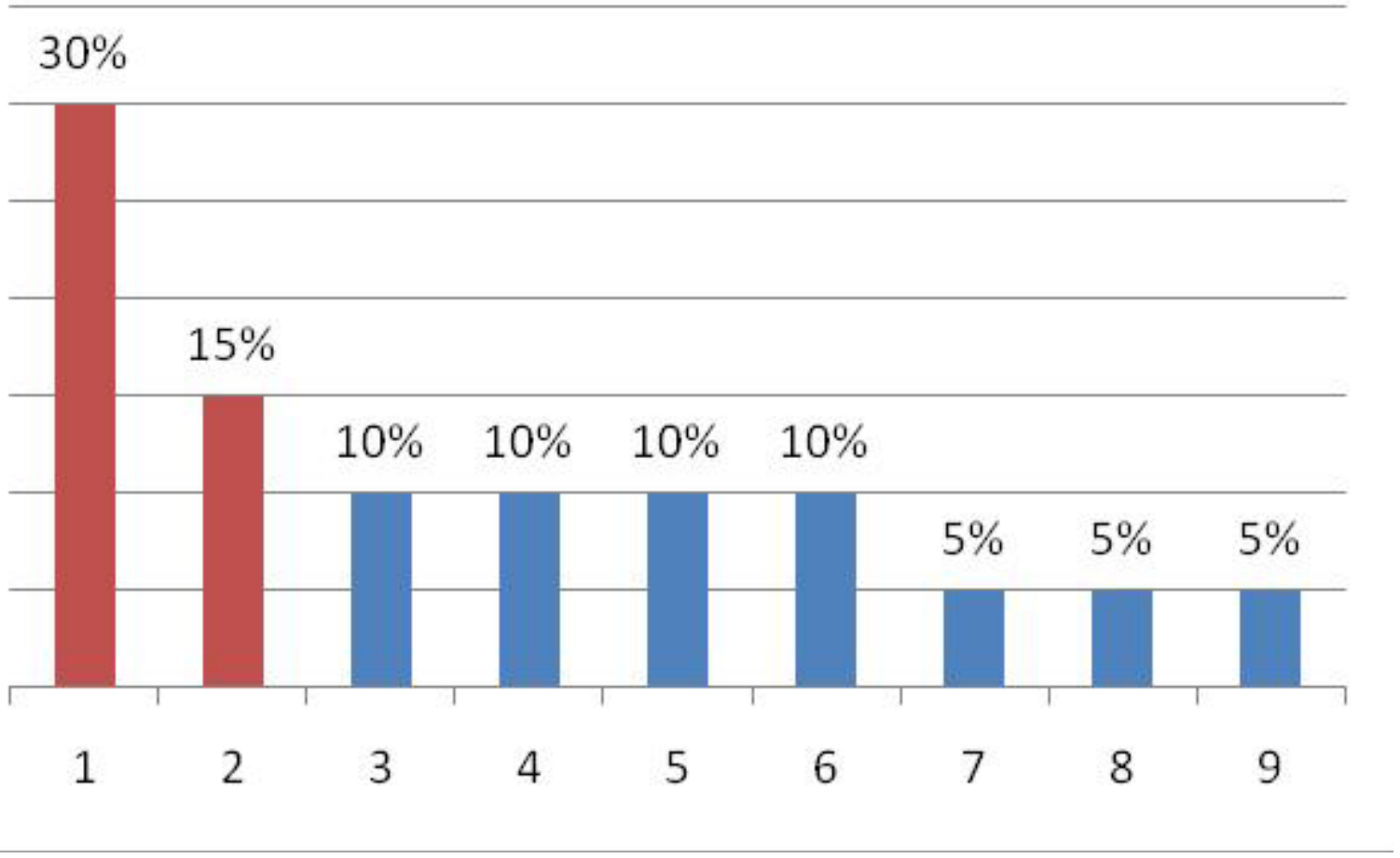

Figura 2. Ocorrência de microorganismos isolados de pacientes durante o processo de implantação das normas preconizadas pela Comissão de Controle de Infecção Hospitalar, setembro a dezembro de 2007, Toledo, Paraná. Total de culturas positivas: 26. 1 = Pseudomonas aeroginosa / sp.; 2 = Klebsiella sp.; 3 = Staphylococcus aureus; 4 = Klebsiella pneumoniae; 5 = Staphylococcus epidermidis; $6=$ Enterobacter cloacae; $7=$ Streptococcus Gama Hemolíticos; 8 = Escherichia coli; $9=$ Enterobacter sp.

Durante a terceira fase de coleta de dados, de dezembro/2007 a março/2008 (figura 3), foram analisados 5 pacientes, com 8 culturas positivas. Com as novas normas da CCIH atuando ativamente, houve uma diminuição significativa no número de pacientes com culturas positivas, assim como na variedade de bactérias presentes nas amostras. A relação entre Staphylococcus epidermidis (10\%) e de Pseudomonas aeruginosa (75\%) também é um resultado das mudanças de atitudes dentro do ambiente hospitalar 


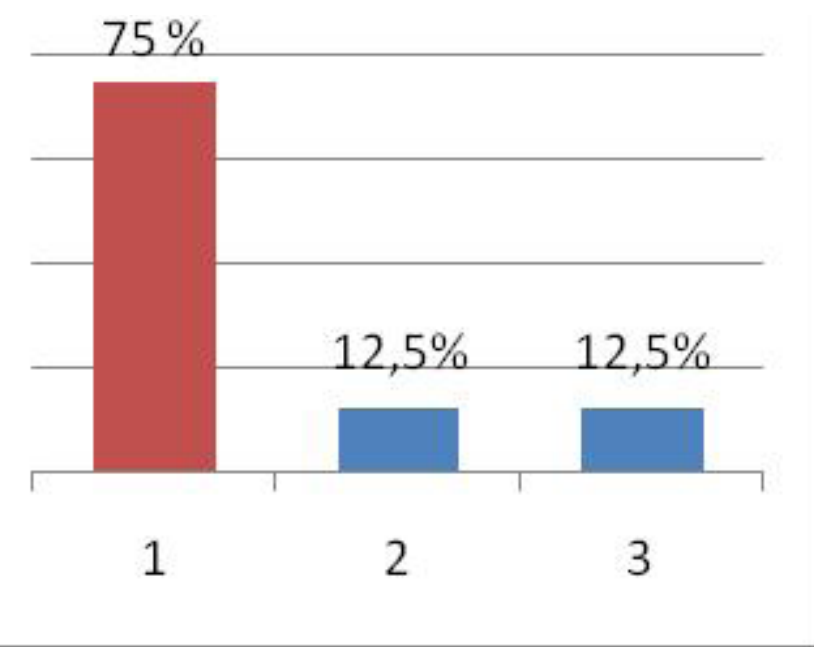

Figura 3. Ocorrência de microorganismos isolados de pacientes após o processo de implantação das normas preconizadas pela Comissão de Controle de Infecção Hospitalar, dezembro/2007 a março/ 2008, Toledo, Paraná. Total de culturas positivas: 8.1 = Pseudomonas aeruginosa/sp.; 2 = Streptococcus Beta Hemolíticos; 3 = Staphylococcus epidermidis.

A respeito da forte evidência da efetividade e eficácia das atividades de vigilância e controle de infecção, não existe estudo que evidencie, precisamente, quais métodos e programas devem ser adotados, na execução da vigilância e controle de infecção hospitalar. A mudança de comportamento, tão necessária neste contexto, não ocorre rapidamente. É necessária a fundamentação prática e teórica e, ainda, a assimilação e aplicação de medidas de prevenção, imperativas à adequada assistência. A formação de hábitos pelos profissionais e, não apenas, a teorização do conhecimento, toma-se em objetivo a ser alcançado pela CCIH (HALEY et al., 1985).

Ao final da quarta fase de coleta de dados (gráfico 4), analisamos seis pacientes com 11 culturas positivas, e observamos, pela primeira vez em aproximadamente um ano de análises, que em nenhum dos onze pacientes com culturas positivas, houve o crescimento de Staphylococcus epidermidis, indicando um resultado mais do que satisfatório após apenas seis meses da implantação da Comissão de Controle de Infecção Hospitalar na Unidade de Terapia Intensiva. A presença de 54,55\% de culturas positivas para Pseudomonas aeruginosas confirma a já comprovada dificuldade de erradicação dessa bactéria multirresistente em ambiente hospitalar e também define esse microorganismo como pertencente a microbiota desta UTI.

Os hospitais, sem um programa de controle de infecção hospitalar estabelecido e atuante, apresentam índices de infecção em torno de $18 \%$, dados que permitem concluir que pelo menos $1 / 3$ das infecções podem ser evitadas (PITTET, 2005; PRADE; OLIVEIRA; RODRIGUES, 1995).

O número de culturas positivas na quarta fase aumentou em relação a terceira fase, devido a um aumento no número de internamentos. As coletas eram feitas de acordo com a avaliação e critério dos médicos, por isso a irregularidade no número de pacientes e dos materiais coletados. 


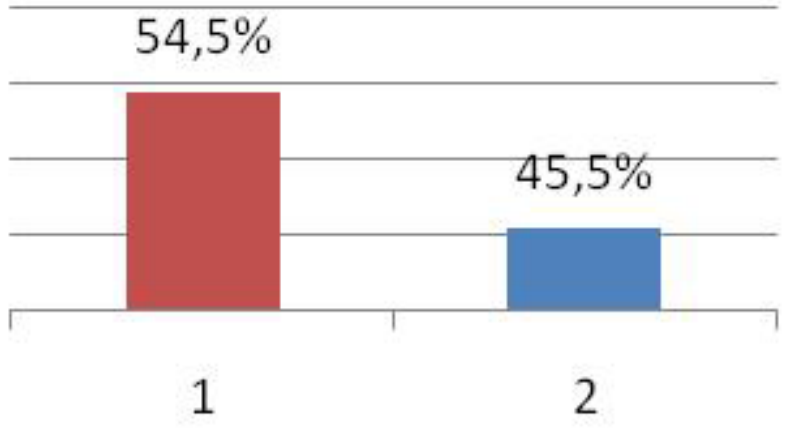

Figura 4. Ocorrência de microorganismos isolados de pacientes aproximadamente um ano após o processo de implantação das normas preconizadas pela Comissão de Controle de Infecção Hospitalar, durante o período de março a junho de 2008, Toledo, Paraná. Total de culturas positivas: 11. 1 = Pseudomonas aeruginosa/sp.; 2 =Enterobacter aerogenes/sp.

\section{Conclusão}

A extinção da infecção hospitalar é uma tarefa inatingível, considerando sua etiologia e condições de instalação no homem em desequilíbrio no seu processo doença. Entretanto, a prevenção e redução têm se mostrado viável em vários casos e situações, conforme já comprovado na prática hospitalar. Conseguimos demonstrar e certificar neste estudo que, com a informação, o treinamento, o comprometimento e a conscientização da equipe médica e colaboradores, quanto à importância do uso diário das normas preconizadas pela Comissão de Controle de Infecção Hospitalar, é possível de se obter resultados significativos e satisfatórios na diminuição dos casos de infecção hospitalar, melhorando a qualidade de assistência à saúde.

\section{Referências}

ANDRADE, D. A.; ANGERAMI, E. L. S. Reflexões acerca das infecções hospitalares às portas do terceiro milênio. Medicina, Ribeirão Preto, v. 32, p. 492-497, 1999.

ANDRADE, D.; LEOPOLDO, V. C.; HAAS, V. J. Ocorrência de bactérias multirresistentes em um Centro de Terapia Intensiva de Hospital Brasileiro de Emergências. Revista Brasileira de Terapia Intensiva, Rio de Janeiro, v. 18, n. 1, p. 31-37, 2006
ASSOCIAÇÃO PAULISTA DE ESTUDOS E CONTROLE DE INFECÇÃO HOSPITALAR. Infecções hospitalares no Brasil: uma medida de sua magnitude nos anos 1990 e comparação com os índices europeus. 2005. Disponível em URL: <http://www.apecih.org.br/ infeccoes_hospitalares.htm>. Acesso em: 3 abr. 2010.

BAUER, A. W.; KIRBY, W. M.; SHERRIS, J. C. Antibiotic susceptibility testing by a standardized single disk method. American Journal Clinical Pathology, Chicago, v. 45, p. 493-496, 1966.

BOYCE, J. M. Coagulase-negative Staphylococci. In: MAYHALL, C.G. (Ed.). Hospital Epidemiology and Infection Control, 2. ed. Philadelphia: Lippincott Williams \& Wilkins, 1999. p. 365-383.

BRASIL. Ministério da Saúde. Curso de introdução ao controle de infecção hospitalar. Brasília, 1985a.

BRASIL. Ministério da Saúde. Manual de controle de infecção hospitalar. Brasília, 1985b.

BRASIL. Ministério da Saúde. Portaria $\mathrm{n}^{\circ}$. 2.616, de 12 de maio de 1998. Dispõe sobre organização e implementação de programas de controle de infecção hospitalar em hospitais. São Paulo, 1998.

BUCHALLA, C. M.; WALDMAN, E. A.; LAURENTI, R. A mortalidade por doenças infecciosas no início e no final do século XX no Município de São Paulo. Revista Brasileira Epidemiologia, São Paulo, v. 6, n. 4: p. 33544. 2003.

CAO, B.; WANG, H.; SUN, H.; ZHU, Y.; CHEN, M. Risk factors and clinical outcomes of noso comial multi-drug resistant Pseudomonas aeruginosa infections. Journal Hospital. Infection, London, v. 57, p. 112-118, 2004. 
CARDO, D. Comparação entre dois métodos de coleta de dados de infecção hospitalar em Hospital de Ensino. 1987. Tese (Mestrado) - Escola Paulista de Medicina, São Paulo.

CAVALCANTI, M.; VALENCIA, M.; TORRES, A. Respiratory nosocomial infections in the medical intensive care unit. Microbes and Infection, Paris, v. 2, p. 292-301, 2005.

CEPED, A. J.; WHITEHOUSE, T.; COOPER, B.; JONES, K.; KWAKU, F. Isolation of patients in single rooms or cohorts to reduce spread of MRSA in intensivecare units: prospective two-centre study. Lancet, Reino Unido, v. 365, p. 295-304, 2005.

COUTO, R. C.; PEDROSA, T. M. G.; NOGUEIRA, J. M. Infecção hospitalar: epidemiologia, controle e tratamento. 3. ed. Rio de Janeiro: Médica e Científica, 2003.

DIENER, J. R.; COUNTINHO, M. S.; ZOCCOLI, C. M. Infecções relacionadas ao cateter venoso central em terapia intensiva. Revista Associação Médica Brasil, São Paulo, v. 42, p. 205-214, 1996.

ÉVORA, Y. D. M.; ALMEIDA, E. C. S. Comissão de controle de infecção hospitalar de um hospital-escola. Revista Paulista de Hospitais, v. 31, n. 3-4, p. 70-73, 1983.

FERNANDES, A. T.; RIBEIRO FILHO, N.; BARROSO, E. A. R. Conceito, cadeia epidemiológica das infecções hospitalares e avaliação custo-benefício das medidas de controle. In: FERNANDES, A. T. (ED.). Infecção hospitalar e suas interfaces na área da Saúde. São Paulo: Atheneu; 2000. p. 215-265.

FERRAZ, E. M. Controle de infecção hospitalar: resultados de um estudo prospectivo de dez anos em um hospital universitário do Recife. 1987. Tese (Disciplina de Técnica Cirúrgica e de Anestesia) - Universidade Federal de Pernambuco, Recife.

FERRAZ, E. M.; FERRAZ, A. A. B.; BACELAR, T. S. D.; ALBUQUERQUE, H. S. T.; VASCONCELOS, M. D. M. M; LEÃO, C. S. Controle de infecção em cirurgia geral: resultado de um estudo prospectivo de 23 anos e 42.274 cirurgias. Revista do Colégio Brasileiro de Cirurgiões, Rio de Janeiro, v. 28, p. 17-26, 2001.

FILE JUNIOR, T. M. Overview of resistence in the 1990s. Chest, Chicago, v. 115, n. 3, p. 3S-8S, 1999.

GALES, A. C.; JONES, R. N.; TURNIDGE, J.; RENNIE, R.; RAMPHAL, R. Characterization of Pseudomonas aeruginosa isolates: occurrence rates, antimicrobial susceptibility patterns, and molecular typing in the global SENTRY antimicrobial surveillance program, 1997-1998. Clinical Infectious Diseases, Oxford, v. 32, s146-s155, 2001.

GIUNTA, A. P. N.; LACERDA, R. A. Inspeção dos programas de controle de infecção hospitalar dos serviços da saúde pela vigilância sanitária: diagnóstico de situação. Revista da Escola de Enfermagem, São Paulo, v. 40, n. 1, p. 64-70, 2006.

GRANSDEN, W. R.; EYKYN, S. J.; PHILLIPS, I. Staphylococcus aureus bacteremia: 400 episodes in St. Thomas's Hospital. British Medical Journal, London, v. 288, p. 300-303, 1984.

HALEY, R. W.; CULVER, D. H.; WHITE, J. W.; MORGAN, W. M.; EMORI, T. G.; MUNN, V. P.; HOOTON, T. M. The Efficacy of Infection Surveillance and Control Programs in Preventing Nosocomial Infections in U. S. Hospitals. American Journal of Epidemiology, Baltimore, v. 121, n. 2, p. 182-205, 1985.

LEVIN, A. S. S.; ARRUDA, E. A. G.; OLIVEIRA, M. S. Infecções por pseudomonas. In: VERONESE R.; FOCACCIA. R. Tratado de infectologia. 3. ed. São Paulo: Atheneu, 2005. p. 971-979.

LEVIN, A. S.; BARONE, A. A.; PENCO, J.; SANTOS, M. V.; MARINHO, I. S.; ARRUDA, E. A. G.; MANRIQUE, E. I.; COSTA, S. F. Intravenous colistin as therapy for nosocomial infections caused by multidrugresistant Pseudomonas aeruginosa and Acinetobacter baumannii. Clinical Infectious Diseases, Oxford, v. 28, p. 1008-1111, 1999.

LIMA, D. A. F. S. Pseudomonas aeruginosa resistente a múltiplos antimicrobianos: avaliação do seu mecanismo de disseminação na Unidade de Emergência do HCFMRP-USP com base na análise de fatores de risco dos pacientes e na genotipagem das amostras. 2006. Tese (Doutorado) - Faculdade de Medicina de Ribeirão Preto da Universidade de São Paulo, Ribeirão Preto.

MICHELIM, L.; LAHUDE, M.; ARAUJO, R. P.; GIOVANAZ, D. S. H.; MULLER, G.; DELAMARE, A. P. L.; COSTA, S. O. P.; ECHEVERRIGARA,Y. S. Pathogenicity factors and antimicrobial resistance of staphylococcus epidermidis associated with nosocomial infections occuring in intensive care units. Brazilian Journal Microbiology, Rio de Janeiro, v. 36, p. 17-23. 2005.

MOREIRA, M.; MEDEIROS, E. A. S.; PIGNATARI, A. C. C.; WEY, S. B.; CARDO, D. M. Efeito da infecção hospitalar da corrente sanguínea por Staphylococcus aureus resistente à oxacilina sobre a letalidade e o tempo de hospitalização. Revista Associação Médica Brasil, São Paulo, v. 44, p. 263-268, 1998.

MYLOTTE, J. M.; McDERMOTT, C.; SPOONER, J. A. Prosrective study of 114 consecutive episodes of 
Staphylococcus. Reviews of infectious disease, Chicago, v. 9, p. 891-907, 1987.

NATIONAL COMMITTEE FOR CLINICAL LABORATORY STANDARDS - NCCLS. Methods for dilution antimicrobial susceptibility tests for bacteria; approved standard. $5^{\text {th }}$. Wayne, Pennsylvania: NCCLS; 2000. Document M7 - A5.

PILONETTO, M.; ROSA, E. A. R.; BROFMAN, P. R. S.; BAGGIO, D.; CALVÁRIO, F. Hospital gowns as a vehicle for bacterial dissemination in an intensive care unit. Brazilian Journal Infectious Diseases, Salvador, v. 8, p. 206-210, 2004.

PITTET, D. Infection control and quality heath care in the new millennium. American Journal Infection Control, St. Louis, v. 33, p. 258-267, 2005.

PRADE, S. S.; OLIVEIRA, S. T.; RODRIGUES, R. Estudo brasileiro da magnitude das infecções hospitalares em hospitais terciários. Revista do Controle de Infecção Hospitalar, v. 2, p. 11-24. 1995.

TURRINI, R. N. T. Infecção hospitalar e mortalidade em hospital pediátrico. 1996. Dissertação (Mestrado em Saúde Pública) - Faculdade de Saúde Pública da Universidade de São Paulo, São Paulo.

VINCENT, J. L. Nosocomial infections in adult intensivecare units. Lancet, Reino Unido, v. 361, p. 2068-2077, 2003.

WATANAKUNAKORN, C.; CHAN, S. J.; DEMARGO, D. G.; PALMER, J. A. Staphylococcus aureus bacteremia: significance of hyperbilirubinemia. Scandinavian journal of infectious diseases, London. v. 19, p. 195-203, 1987.

WOJKOWSKA-MACH, J; BULANDA, M.; ROZANSKA, A.; KOCHAN, P.; HECZKO, P. B. Hospital-acquired pneumonia in the intensive care units of Polish hospitals. Infection control and hospital epidemiology, Chicago, v. 27, n. 7, p. 784-786, 2006.

ZAVASCKI, A. P.; CRUZ, R. P.; GOLDANI, L. Z. Risk factors for imipenem-resistant Pseudomonas aeruginosa: a comparative analysis of two case-control studies in hospitalized patients. Infection control and hospital epidemiology, Thorofare, v. 59, n. 2, p. 96-101, 2005.

Recebido em 23 de novembro de 2009 - Received on November 23, 2009

Aceito em 22 de outubro de 2010 - Accepted on October 22, 2010 
